Abstract THU0234 -Table 1.

\begin{tabular}{|c|c|c|c|c|c|c|c|c|c|c|c|c|c|}
\hline & & IKAROS & AIOLOS & $\mathrm{CD} 3$ & $\mathrm{CD} 20$ & CD138 & FOCI & $\operatorname{IgA}$ & $\operatorname{IgG}$ & $\operatorname{IgM}$ & C3 & $\mathrm{C} 4$ & ESDDAI \\
\hline Spearman's & IKAROS & 1.000 & $.511^{-*}$ & $477^{* *+}$ & .453" & .150 & $.417^{*}$ & .053 & $.337^{*}$ & .012 & -.137 & -293 & $.440^{*}$ \\
\hline rho & AIOLOS & $.511^{*}$ & 1.000 & $.577^{* *}$ & $.370^{\circ}$ & .256 & $.514^{*-}$ & -.019 & .160 & .052 & .110 & .092 & .286 \\
\hline
\end{tabular}

Results: The density of Ikaros and Aiolos positive cells was significantly higher in the SGs of ELS positive SS patients compared to NSCS. As shown in Table 1, the density of Ikaros and Aiolos positive cells in the SG of SS patients significantly correlated with $B$ and $T$ cell semiquantitative scores and the number of lymphocytic foci. Ikaros positive cell density also correlated with $\lg$ levels and disease activity measured by ESSDAI. Accordingly, SS patients with high expression of Ikaros ( $>10$ Ikaros $^{+}$cells $\left./ \mathrm{mm}^{2}, n=11 / 23\right)$ had significantly higher ESSDAI $(p=0.023)$ and higher prevalence of ANA positivity $(p=0.019)$. In vitro, iberdomide significantly inhibited the TLR7-mediated production of IgG from Sjögren's $B$ cells $(p=0.03)$ and significantly reduced the number of $C D 27^{+} C D 38^{+}$ plasmablasts $(p=0.03)$

Conclusion: We here show that the transcription factors Ikaros and Aiolos are expressed in the salivary glands of patients with Sjögren's syndrome with ELS. The association of Ikaros with disease severity and autoantibody positivity and the inhibition of B cell activation by iberdomide point to the relevance of these transcription factors in Sjögren's, thus suggesting their potential use as therapeutic targets in patients with Sjögren's and other B cell-mediated autoimmune diseases.

Acknowledgement: This study was supported by research funding from Celgene Corporation.

Disclosure of Interests: Felice Rivellese: None declared, Sotiria ManouStathopoulou: None declared, Daniele Mauro: None declared, Elena Pontarini: None declared, Davide Lucchesi: None declared, MATTIA CONGIA: None declared, Peter Schafer Shareholder of: Celgene corporation, Employee of: Celgene Corporation, Nurhan Sutcliffe: None declared, Costantino Pitzalis Grant/research support from: Celgene, Myles Lewis Grant/ research support from: Celgene, Michele Bombardieri Grant/research support from: Celgene, Consultant for: Medimmune

DOI: 10.1136/annrheumdis-2019-eular.7333

\section{THU0235 INHIBITION OF EZH2 AMELIORATES LUPUS-LIKE DISEASE IN MRL/LPR MICE}

Dallas Rohraff ${ }^{1}, \mathrm{Ye} \mathrm{He}^{1,2}$, Evan Farkash ${ }^{1}$, Mark Schonfeld ${ }^{1}$, Eliza Tsou ${ }^{1}$, Amr Sawalha ${ }^{1,3}$. ${ }^{1}$ University of Michigan, Ann Arbor, United States of America; ${ }^{2}$ The Second Xiangya Hospital, Central South University, Changsha, China;

${ }^{3}$ University of Pittsburgh, Pittsburgh, United States of America

Background: We previously revealed a role for $\mathrm{EZH} 2$ in inducing proinflammatory epigenetic changes in lupus CD4+ T cells.

Objectives: In this study, we sought to determine if inhibiting EZH2 ameliorates lupus-like disease in MRL/lpr mice.

Methods: EZH2 expression levels in multiple cell types in lupus patients were evaluated using flow cytometry and mRNA expression data. Inhibition of $\mathrm{EZH} 2$ in $\mathrm{MRL} / \mathrm{lpr}$ mice was achieved by DZNep intraperitoneal administration using a preventative and a therapeutic treatment model. Effects of DZNep on animal survival, anti-dsDNA antibody production, proteinuria, renal histopathology, cytokine production, and $T$ and $B$ cell numbers and percentages were assessed.

Results: EZH2 expression levels were increased in whole blood, neutrophils, monocytes, B cells, and CD4+ T cells in lupus patients. In MRL/lpr mice, inhibiting EZH2 with DZNep treatment before or after disease onset improved survival and significantly reduced anti-dsDNA antibody production. DZNep-treated mice displayed a significant reduction in renal involvement, splenomegaly, and lymphadenopathy. Lymphoproliferation and numbers of double-negative $T$ cells were significantly reduced in DZNep treated mice. Concentrations of circulating cytokines and chemokines, including TNF, IFN- $\gamma$, CCL2, RANTES/CCL5, IL-10, KC/CXCL1, IL-12, IL$12 \mathrm{p} 40$ and MIP-1//CCL4 were decreased in DZNep treated mice.

Conclusion: $\mathrm{EZH} 2$ is upregulated in multiple cell types in lupus patients. Therapeutic inhibition of $\mathrm{EZH} 2$ abrogates lupus-like disease in $\mathrm{MRL} / \mathrm{lpr}$ mice, suggesting that EZH2 inhibitors may be repurposed as a novel therapeutic option in lupus patients.

Disclosure of Interests: None declared

DOI: 10.1136/annrheumdis-2019-eular.2503
THU0236

URINARY EXOSOMAL MIR-31, MIR-107 AND MIR-135B5P FROM TUBULAR RENAL CELLS AS RESPONDER BIOMARKER IN LUPUS NEPHRITIS

Cristina Solé-Marcé ${ }^{1}$, Eloi Garcia-Vives ${ }^{1}$, Irene Agraz ${ }^{2}$, Josep Ordi-Ros ${ }^{1}$, Josefina Cortés-Hernández'. ${ }^{1}$ Vall Hebron Institute Research, Internal Medicine Lupus Unit, Barcelona, Spain; ${ }^{2}$ Hospital Vall Hebron, Department of Renal Pathology, Barcelona, Spain

Background: Lupus nephritis (LN), occurring in $40-75 \%$ of patients with systemic lupus erythematosus (SLE), continues to be one of the most severe forms of lupus with an unpredictable course. Conventional clinical parameters are not sensitive or specific enough for detecting ongoing disease activity, early relapse, disease progression or response to therapy [1]. Exosomal-derived urinary miRNAs can accurately reflect renal dysfunction and structural damage making them good biomarkers for diagnosis and prognosis [2]. In LN, urinary exosomal miR-29c expression levels have been identified as predictor of early fibrosis [3] and miR-146a upregulation as marker to discriminate active LN [4]. To the best of our knowledge, this is the first screening of miRNAs in urinary exosomes from LN patients to study their role as predictors of response to therapy. Objectives: Identify a non-invasive miRNA profile predictive of clinical response in $L N$ following standard treatment.

Methods: Urinary exosomes were isolated and characterized from lupus patients $(\mathrm{N}=14)$ during flare and post-treatment time. Patients were divided between responder and non-responder and urinary exosomal miRNA screening analysis was performed according their clinical response. To validate initial results, a new cohort of $\mathrm{LN}$ patients were used $(\mathrm{N}=44)$. In situ hybridization of miRNAs in kidney renal biopsies and in vitro experiments with human primary renal cells were performed in order to determinate cell origin of exosome production and their delivery cell target (Figure 1)

Results: In the screening, 25 miRNAs were significantly differences in comparative group analysis. High levels of miR-31, miR-107 and miR $135 b-5 p$ were confirmed to be related with responder patients. We found that miR-135b has the best profile to distinguish the two groups (AUC= $0.783(95 \%$ confidence interval $[\mathrm{Cl}], 0.640-0.926)$, cut-off $<0.0884$ with $77.8 \%$ sensitivity and $71.4 \%$ specificity). Renal tissue samples from responder patients showed miR-31, miR-107 and miR-135b-5p to be highly expressed compared with non-responders. All miRNAs were predominantly localized in the tubular cells. Stimulated tubular renal cells displayed the higher expression levels of exosome-derived miR-31, miR-107 and miR$135 b-5 p$ when compared with endothelial or mesangial cells $(p<0.0001)$ Nevertheless, urinary exosomes from non-responder patient's internalization was only of $50 \%$ by mesangial cells compared to $90 \%$ from responders.

Conclusion: These results indicated that levels of urinary exosomal miRNAs produced by tubular cells might have a renal recovery role in mesangial cells and be used as new biomarkers of lupus nephritis outcome.

\section{REFERENCES:}

[1] Adler M, et al. Rheumatology. 2006;45(9):1144-7.

[2] Valadi H, et al. Nat Cell Biol. 2007;9(6):654-U672.

[3] Solé C, et al. Neprol Dial Transplant. 2015. 30(9):1488-96.

[4] Perez-Hernandez J, et al. PLoS One. 2015 10(9):e0138618.

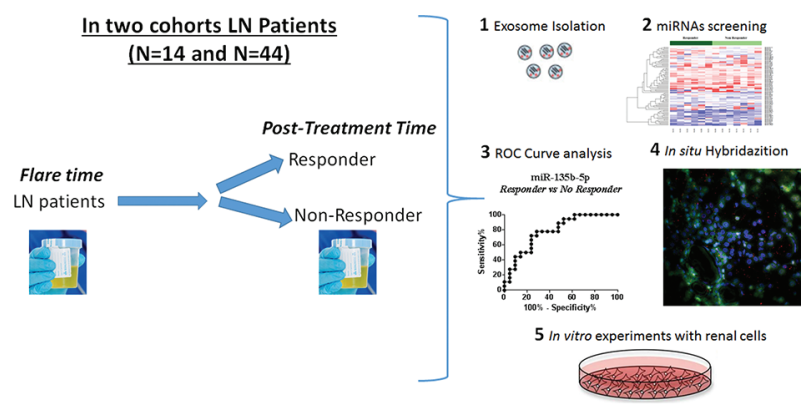

Abstract THU0236 - Figure 1. Methodology Scheme. Urine from two cohorts of LN patients to isolate exosomes, perform miRNAs screening, ROC curve analysis, in situ hybridazition and in vitro experiments.

Acknowledgement: This work was supported by grants from the Spain Government (Instituto de Salud Carlos III, PI15/02117) and the Catalan Lupus Foundation. The funders of this study were not involved in study 